\title{
On the interaction of pulsar winds and clumpy stellar winds
}

\author{
Kefala E.* \\ E-mail: ekefalke7@alumnes.ub.edu
}

\section{Bosch-Ramon V.}

Departament de Física Quàntica i Astrofísica, Institut de Ciències del Cosmos (ICCUB),

Universitat de Barcelona (IEEC-UB), Martí i Franquès 1, 08028 Barcelona, Spain

E-mail: vboschefqa.ub.edu

\begin{abstract}
High-mass binaries hosting a non-accreting pulsar are sources of gamma rays, which are thought to originate in the wind-wind collision region. The stellar wind is expected to be clumpy, which could have important effects on the emitting flow dynamics, and thus on the associated nonthermal radiation. We adopt here a combination of Monte-Carlo and semi-analytical hydrodynamic calculations to explore the impact of clumps on the shocked wind structure on the scales of the binary system.
\end{abstract}

High Energy Phenomena in Relativistic Outflows VII - HEPRO VII

9-12 July 2019

Facultat de Física, Universitat de Barcelona, Spain

\footnotetext{
* Speaker.
} 


\section{Introduction}

The recently established class of gamma-ray binaries comprises a small but growing number of powerful galactic sources. One defining characteristic is the broad non-thermal emission that is dominated by the gamma-ray domain. Few such systems have been detected at high energies (100 $\mathrm{MeV}-100 \mathrm{GeV}$ ) and/or at very high energies (>100 GeV) [1], and several possible candidates are yet to be confirmed (e.g. [2]). The optical companions of the brightest objects, i.e. the most luminous of the canonical gamma-ray binaries, are massive OB-type stars, whereas the vast majority of compact objects in these systems remain unidentified. The detection of radio pulsations in the binary PSR B1259-63/LS 2883 [3] points to a rotation-powered pulsar, basically confirming the hypothesis that the high-energy emission is powered by the pulsar spin-down. The emitting region is thought to be the shock front formed by the collision of the stellar and pulsar winds $[4,5]$. Although an accretion-ejection framework cannot be yet fully discarded, the young pulsar scenario is typically invoked to explain the gamma-ray binaries with unknown compact object (e.g. [6]).

In the young pulsar scenario the bulk of the non-thermal emission originates in the stellar and pulsar wind interaction region. Therefore, it is mandatory to properly understand the properties and dynamics of the colliding flows before modeling the non-thermal radiation from that region. A very relevant issue is the density structure and degree of inhomogeneity of stellar winds, as radiative instabilities in the formation zone of the stellar wind are expected to lead to the formation of clumps $[7,8,9]$. These clumps could potentially distort the two-wind interaction structure, and significantly effect the radiative output [10]. The situation cannot be compared to that of two massive star winds colliding [11], as a relativistic pulsar wind will deposit much more energy on each clump than another stellar wind.

To study the impact of a clump on the colliding wind region, relativistic, axisymmetric, hydrodynamical (RHD) simulations were carried out by Paredes-Fortuny et al. (2015) [12] to study in more detail the impact of differently-sized clumps on the two-wind interaction region. Based on this work, de la Cita et al. (2017) [13] showed that when stellar wind clumps perturb the twowind interaction region, the radiative output of the associated non-thermal radiation can also be largely affected. Similarly, the presence of clumps in the stellar wind could impact the gamma-ray emission powered by microquasar jets [14, 15, 16, 17].

Numerical simulations of the whole interaction region are very expensive, and are not versatile enough if a large parameter space is too be studied. Therefore, it can be useful to devise a simplified method, which nevertheless includes some relevant features of the physical scenario, to model the clumpy-pulsar wind interaction. In this work, we look at the dynamical evolution of multiple clumps under the impact of the pulsar wind using a Monte Carlo method for clumpy wind launching, and a semi-analytical hydrodynamic model for clump evolution. With this method we intend to derive some semi-quantitative conclusions about the properties of the emitting flow ascribed to the wind-wind interaction structure on the binary scales (i.e. without considering orbital motion), which should be affected by the arrival of clumps to some extent.

\section{Numerical approach}

The modelled scenario consists of a galactic binary system hosting a massive star of $R_{*}=10 R_{\odot}$ 
and a non-accreting pulsar at a separation distance of $d=3 \times 10^{12} \mathrm{~cm}$. The massive star powers a supersonic, radiation-driven wind that interacts with the powerful, relativistic, homogeneous wind of the pulsar. The considered stellar wind is however assumed, in the region of interest, to be already highly inhomogeneous, with the entirety of the mass-loss assumed to be carried by clumps at a rate of $\dot{M}=10^{-7} M_{\odot} \mathrm{yr}^{-1}$. The latter assumption is reasonable if the clump-to-inter-clump density ratio is well below the clump-to-average wind ratio $\chi^{2 / 3}$, so then it as a negligible ram pressure. The clumps are assumed to be spherical and are isotropically launched from the stellar surface. The pulsar wind parameters are the bulk Lorentz factor $\Gamma_{\mathrm{pw}}=10^{5}[18,19]$ and power $L_{\mathrm{pw}}=3 \times 10^{36} \mathrm{erg} \mathrm{s}^{-1}$. The system parameters we have used for the two winds are typical for gamma-ray emitting binaries (e.g. [6]).

\subsection{Hydrodynamical model}

As the stellar and pulsar winds collide, they form two bow-like shocks, and two regions in between filled with the respective shocked winds separated by a contact discontinuity (CD). We have assumed that these two regions are very thin, i.e. the geometry of the $\mathrm{CD}$ is representative of the geometry of the whole structure. The $\mathrm{CD}$ is computed through a simple numerical approximation that assumes that it is still mostly shaped by the average effect of the clumps as they are shocked and disrupted by the pulsar wind. Such an assumption is reasonable as long as the clumps cannot sustain for long the dynamical impact of the pulsar wind, which we test and found to be the case for indivitual clump-pulsar wind interactions. Assuming that $r$ and $r_{\mathrm{p}}$ correspond to the clump separation distance from the star and the pulsar, respectively, the equation for the $\mathrm{CD}$ is given by [20]:

$$
\frac{d x}{d y}=\frac{1}{y}\left(x-\frac{d r^{2}(x, y) \sqrt{\eta(x, y)}}{r^{2} \sqrt{\eta(x, y)}+r_{\mathrm{p}}^{2}(x, y)}\right),
$$

where $\eta(x, y)=\left(L_{\mathrm{pw}} / c\right) / \dot{M} / u_{\mathrm{sw}}$ is the local pulsar-to-stellar wind momentum rate ratio, and $u_{\mathrm{sw}}$ the stellar wind velocity. The boundary condition $d x /\left.d y\right|_{y \rightarrow 0} \rightarrow 0$ is the location of the stagnation point along the binary axis where the two winds balance $x_{0}=d /\left(1+\sqrt{\eta_{\infty}}\right)$. This assumes that the stellar wind has reached its terminal velocity already at the $\mathrm{CD} u_{\infty}=2 \times 10^{8} \mathrm{cms}^{-1}$, which is true in fact only if $d \gg R_{*}$.

The clumps are launched anisotropically from the stellar surface, and move ballistically until some of them reach the $\mathrm{CD}$, beyond which the clumps start to be affected by the pulsar wind. Assuming that the only force exerted on the clumps is the pulsar wind ram pressure, $P_{\mathrm{pw}}=L_{\mathrm{pw}} / 4 \pi c r_{p}^{2}$, and considering only individual clump evolution as independent from other clumps or the $\mathrm{CD}$, the clump equation of motion in the radial direction with respect to the pulsar can be described as (based on the expression derived by [21] for jet-cloud interactions):

$$
\frac{d \Gamma_{c}}{d t}=\left(\frac{1}{\Gamma_{c}^{2}}-\frac{\Gamma_{c}^{2}}{\Gamma_{\mathrm{pw}}^{4}}\right) \frac{\pi R_{\mathrm{c}}^{2}}{4 M_{c} c^{2}} P_{\mathrm{pw}} .
$$

This approximation is valid as long as the gravity force is much smaller than the pulsar wind ram pressure force, which is true for clumps moving much faster than the local escape velocity, and well outside the pulsar Bondi radius. The radiation pressure of the star is also a second order effect, 
but should be taken into account in very bright and compact systems. This issue is related to the validity of the assumption that the wind moves already at its maximum speed.

As a result of the effect of the pulsar wind ram pressure shocking the clump, the shocked clump material starts to expand at the local sound speed $c_{\mathrm{s}}=\sqrt{\hat{\gamma} P_{\mathrm{pw}} / h \rho_{\mathrm{c}}}$, where $h=1+\frac{\hat{\gamma}}{\hat{\gamma}-1} \frac{P_{\mathrm{pw}}}{\rho_{\mathrm{c}} c^{2}}$ is the internal specific enthalpy normalized by $c^{2}, \rho_{\mathrm{c}}$ the clump density, and $\hat{\gamma}=5 / 3$ the adiabatic index.

In order to restrict the clump growth, we only let the clump expansion last for the duration of the typical expansion timescale, $t_{\exp }=R_{\mathrm{c}} / c_{\mathrm{s}}$, after crossing the $\mathrm{CD}$, as this is also the typical life span of the shocked clumps before they get disrupted (e.g. [12]). The clumps eventually leave the computational grid, and/or get deflected back to the wind-wind interacting region, where the clump material gets confined by the pulsar and stellar wind ram pressures (through the newlyarriving unshocked clumps). The disrupted clumps quickly get integrated in the shocked material there [12].

\subsection{Results}

We simulated a total of four cases to account for different clump distributions of clump masses and sizes. First, we considered two clump-to-average wind density ratios, $\chi=10$ and $\chi=100$, to account for lighter and heavier clumps (for the same clump size), respectively. Then, keeping a fixed density ratio, we introduced two power-law distributions per clump mass (i.e. clump number per mass unit) with indexes $k=2$ and $k=3$. The minimum and maximum values for the clump mass correspond to $R_{\mathrm{c}, \min }=10^{10} \mathrm{~cm}$ and $R_{\mathrm{c}, \max }=10^{11} \mathrm{~cm}$, respectively. The results shown below are preliminary and are intended to outline the dynamical implications of a clumpy stellar wind.

In Fig. 1, we present snapshots of 2-dimensional (2D) cuts on the orbital plane for the different stellar wind models explored. For a rough estimate of the clump filling factor, we also include 3D images of the computational grid in Fig. 2. As expected, the case shown in Figs. 1c and 2c is the closest to the smooth wind scenario, although the clumps may still significantly perturb the shocked structure. When larger and/or heavier clumps are expected with higher probability, the clumps can penetrate deeper towards the pulsar and strongly distort the interaction region, as in the more extreme case of Fig. $1 b$ and $2 b$.

Clump expansion is an important factor here and largely depends on the initial clump parameters. Lighter, less dense clumps are more easily pushed backwards and get rapidly confined in the interaction region by the two winds and the surrounding material. Denser clumps, on the other hand, generally reach much closer to the pulsar, where the interaction is stronger and their expansion is accelerated. For the most significant events, the subsequent compression of the termination shock of the pulsar wind could lead to changes of the non-thermal activity on a time-scale of minutes or hours, since the clump expansion timescale ranges between $5 \times 10^{2} \mathrm{~s}$ to $5 \times 10^{3} \mathrm{~s}$.

\section{Discussion}

The dynamical impact of the wind clumpiness on the shape and stability of the overall interaction structure is determined, to a greater or lesser extent, by the wind parameters, represented in our prescription by the filling factor, and the clump mass distribution. Nevertheless, our semiquantitative results already show that structured stellar winds should generate spatial and tempo- 


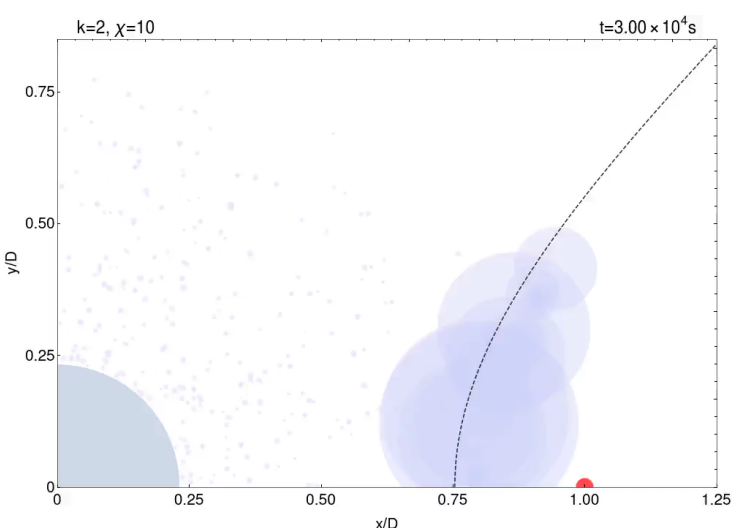

(a)

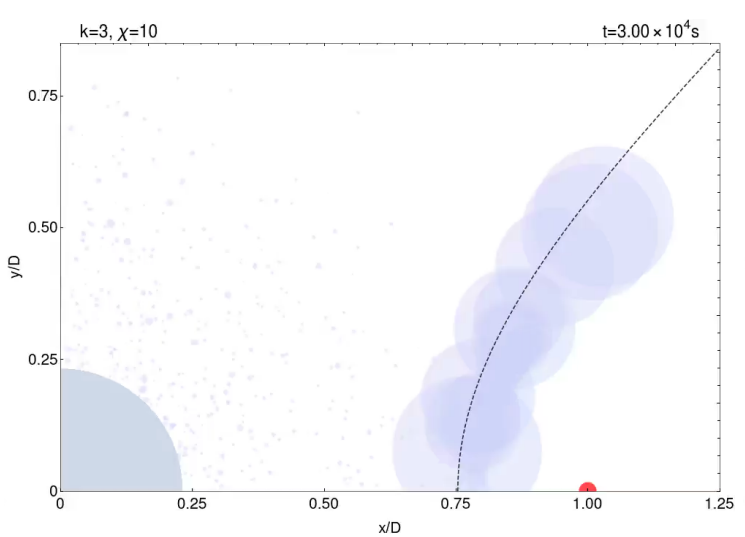

(c)

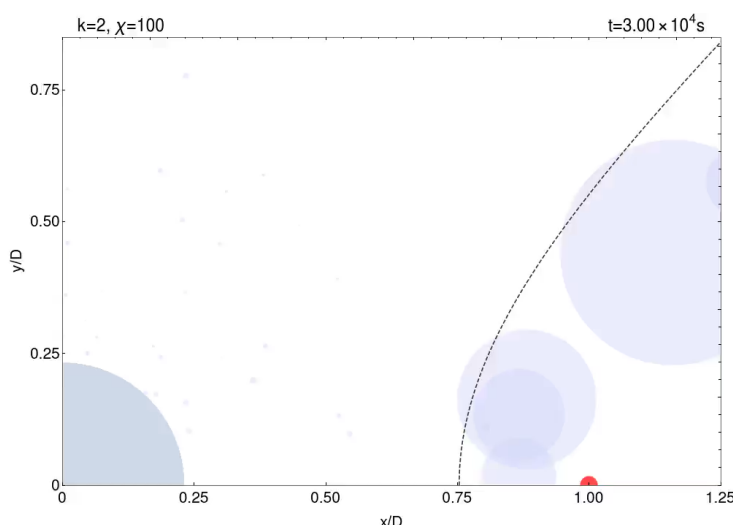

(b)

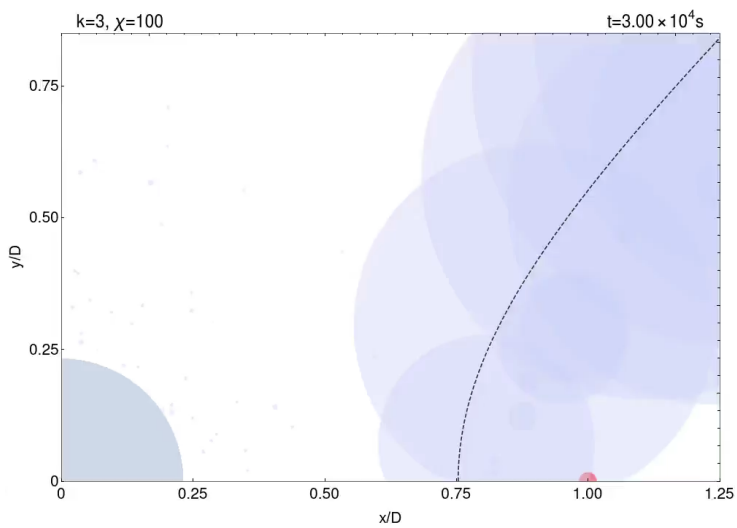

(d)

Figure 1: $2 \mathrm{D}$ cuts of the orbital plane for the four different configurations at $t=3 \times 10^{4} \mathrm{~s}$ : (a) $k=2, \chi=10$, (b) $k=2, \chi=100$, (c) $k=3, \chi=10$, and (d) $k=3, \chi=100$. The solid blue and red surfaces represent the star (left) and pulsar (right; not to scale) that are fixed at a distance $d=3 \times 10^{12} \mathrm{~cm}$. The dashed line gives the shape of the CD.

ral irregularities in the two-wind interaction region of gamma-ray binaries: In all the simulated cases, at least some clumps manage cross the contact discontinuity, and the rare most massive clumps should strongly temporarily modify the shocked wind structure. Therefore, we find that the CD should strongly fluctuate with the compression of the shocked pulsar wind and the arrival of different mass clumps. As the shocked structure is unstable even for smooth stellar winds (e.g. $[22,23,12])$, the arrival of the smallest clumps should at least enhance instability growth even further.

Given the above findings, the implications on the non-thermal flow emission should be prominent: likely enhancement of energy dissipation that goes to non-thermal particles (e.g. through additional shocks and turbulence), and increased synchrotron cooling rates when large clumps reduce the colliding-wind region. The latter effect is related to an amplification of the magnetic field, directly affecting the synchrotron losses that dominate the $\mathrm{X}$-ray band. When the emitting region is largely reduced, gamma-ray emission can also be affected as a result of synchrotron self-Compton 


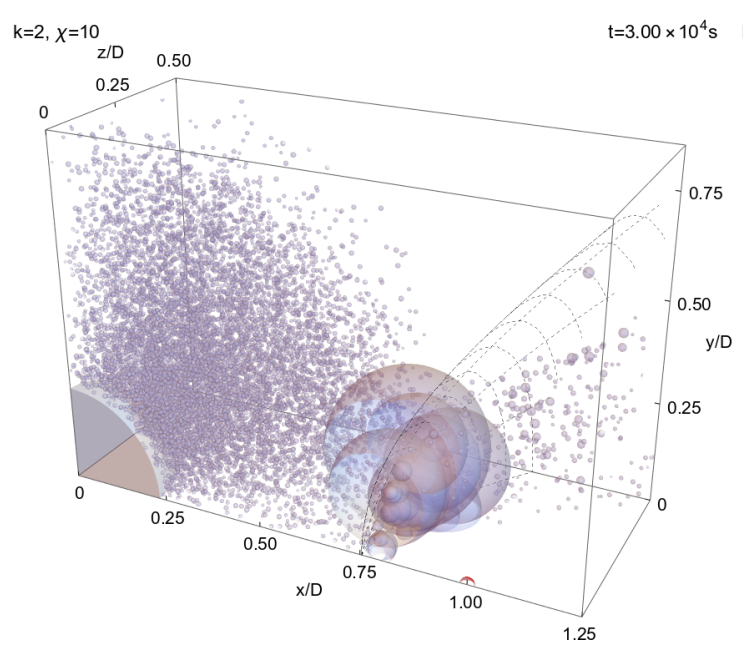

(a)

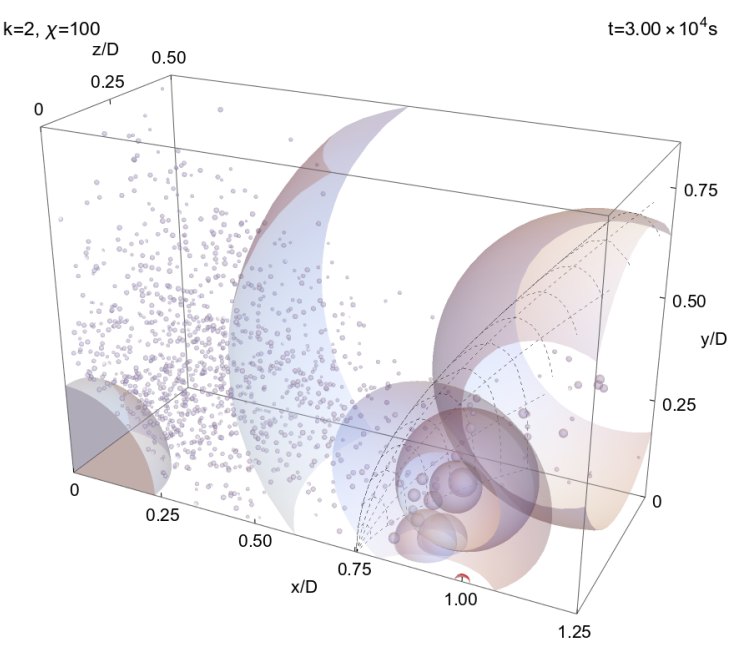

(b)

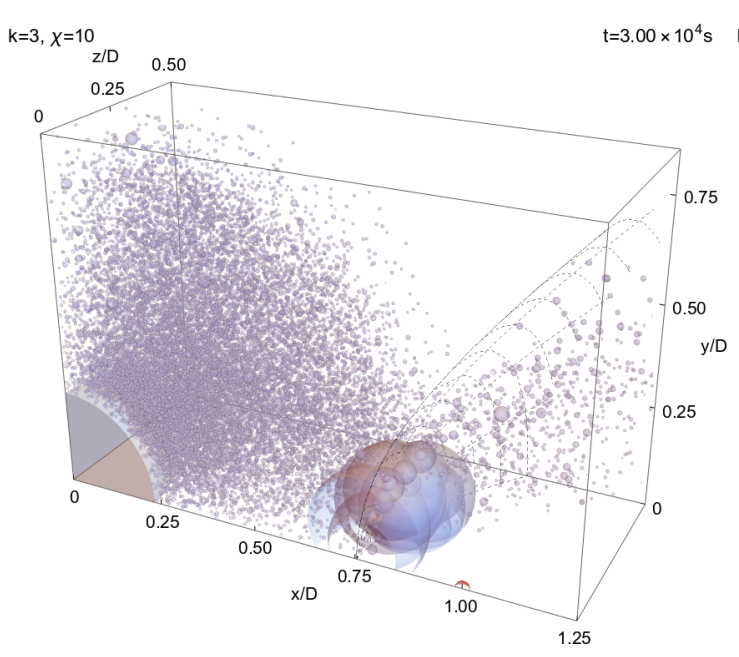

(c)

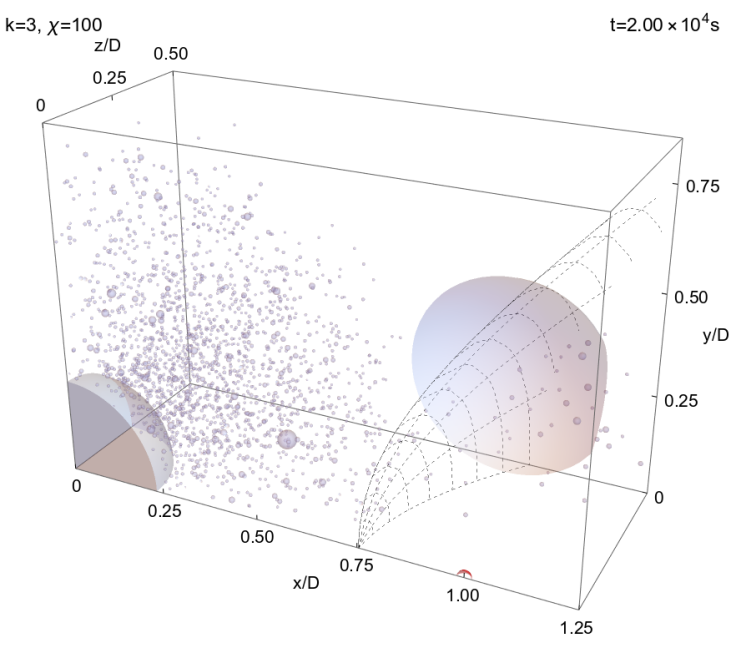

(d)

Figure 2: Images of the computational grid in 3D for the four different configurations at $t=3 \times$ $10^{4}$ s: (a) $k=2, \chi=10$, (b) $k=2, \chi=100$, (c) $k=3, \chi=10$, and (d) $k=3, \chi=100$. The stationary blue and red objects represent the star and pulsar (not to scale) that are fixed at a distance $d=3 \times 10^{12} \mathrm{~cm}$. The dashed mesh is a representation of the CD in 3D.

and intrinsic $\gamma \gamma$ absorption. Therefore, the clumpy stellar wind scenario has been proposed to explain the observed gamma-ray flares of PSR B1259-63/LS 2883 [12], and the quick X-ray variability (e.g. LS 5039, LS I +61 303) in high-mass binaries hosting compact objects [10]. The effects of Doppler boosting combined with the strongly perturbed velocity field could also be important, producing complex radiative patterns both in time and beaming [13].

The implementation of a simplified treatment using Monte Carlo simulations allows for an estimate of the frequency at which clumps interact with the shocked pulsar wind. Then, radiation computations can be made not only for each clump individually, but also for multiple events collectively. It also allows for quick tracking of the temporal evolution of the SEDs and light-curves in 
correlation with the flow dynamics. In future work, a radiation prescription will be developed. Furthermore, we plan to quantify the approximate rates of the most significant events for the explored cases. Also, a more realistic expansion model should be eventually adopted to more accurately limit the clump growth. Finally, one can improve as well the dynamical model by adding the radiation pressure of the stellar wind, the role of the system gravity fields, clump-clump interactions, and considering the role of the inter-clump medium. Eventually, however, a numerical study akin to [16], but in the context of high-mass binaries with a young pulsar should be carried out.

\section{Acknowledgements}

This work was supported by the Spanish Ministerio de Economía y Competitividad (MINECO/FEDER, UE) under grant AYA2016-76012-C3-1-P with partial support by the European Regional Development Fund (ERDF/FEDER), MDM-2014-0369 of ICCUB (Unidad de Excelencia 'María de Maeztu'), and the Catalan DEC grant 2017 SGR 643.

\section{References}

[1] J. M. Paredes and P. Bordas, Phenomenology of gamma-ray emitting binaries, arXiv e-prints (2019) arXiv:1902.09898 [1902.09898].

[2] P. Eger, H. Laffon, P. Bordas, E. de Oña Whilhelmi, J. Hinton and G. Pühlhofer, Discovery of a variable X-ray counterpart to HESS J1832-093: a new gamma-ray binary?, MNRAS 457 (2016) 1753 [1601.03208].

[3] S. Johnston, R. N. Manchester, A. G. Lyne, M. Bailes, V. M. Kaspi, G. Qiao et al., PSR 1259-63: A Binary Radio Pulsar with a Be Star Companion, ApJl 387 (1992) L37.

[4] L. Maraschi and A. Treves, A model for LS I +61 303., MNRAS 194 (1981) 1P.

[5] M. Tavani and J. Arons, Theory of High-Energy Emission from the Pulsar/Be Star System PSR 1259-63. I. Radiation Mechanisms and Interaction Geometry, ApJ 477 (1997) 439 [astro-ph/9609086].

[6] G. Dubus, Gamma-ray binaries and related systems, A\&Ar 21 (2013) 64 [1307. 7083 ].

[7] L. B. Lucy and P. M. Solomon, Mass Loss by Hot Stars, ApJ 159 (1970) 879.

[8] M. C. Runacres and S. P. Owocki, The outer evolution of instability-generated structure in radiatively driven stellar winds, A\&A 381 (2002) 1015.

[9] J. Puls, J. S. Vink and F. Najarro, Mass loss from hot massive stars, A\&Ar 16 (2008) 209 [0811.0487].

[10] V. Bosch-Ramon, Clumpy stellar winds and high-energy emission in high-mass binaries hosting a young pulsar, A\&A 560 (2013) A32 [1310.5641].

[11] J. M. Pittard, A Clumping-independent Diagnostic of Stellar Mass-Loss Rates: Rapid Clump Destruction in Adiabatic Colliding Winds, ApJl 660 (2007) L141 [astro-ph/ 0703617 ].

[12] X. Paredes-Fortuny, V. Bosch-Ramon, M. Perucho and M. Ribó, Simulations of an inhomogeneous stellar wind interacting with a pulsar wind in a binary system, A\&A 574 (2015) A77 [1411. 3593]. 
[13] V. M. de la Cita, S. del Palacio, V. Bosch-Ramon, X. Paredes-Fortuny, G. E. Romero and D. Khangulyan, Gamma rays from clumpy wind-jet interactions in high-mass microquasars, A\&A 604 (2017) A39 [1701.05028].

[14] S. P. Owocki, G. E. Romero, R. H. D. Townsend and A. T. Araudo, Gamma-Ray Variability from Wind Clumping in High-Mass X-Ray Binaries with Jets, ApJ 696 (2009) 690 [0902 . 2278].

[15] A. T. Araudo, V. Bosch-Ramon and G. E. Romero, High-energy emission from jet-clump interactions in microquasars, A\&A $\mathbf{5 0 3}$ (2009) 673 [0906.4803].

[16] M. Perucho and V. Bosch-Ramon, 3D simulations of microquasar jets in clumpy stellar winds, A\&A 539 (2012) A57 [1112.2520].

[17] V. M. de la Cita, S. del Palacio, V. Bosch-Ramon, X. Paredes-Fortuny, G. E. Romero and D. Khangulyan, Gamma rays from clumpy wind-jet interactions in high-mass microquasars, Astronomy \& Astrophysics 604 (2017) A39.

[18] D. Khangulyan, F. A. Aharonian, S. V. Bogovalov and M. Ribó, Post-periastron Gamma-Ray Flare from PSR B1259-63/LS 2883 as a Result of Comptonization of the Cold Pulsar Wind, ApJl 752 (2012) L17 [1107. 4833$]$.

[19] F. A. Aharonian, S. V. Bogovalov and D. Khangulyan, Abrupt acceleration of a 'cold' ultrarelativistic wind from the Crab pulsar, Nature 482 (2012) 507.

[20] I. I. Antokhin, S. P. Owocki and J. C. Brown, A Steady, Radiative-Shock Method for Computing X-Ray Emission from Colliding Stellar Winds in Close, Massive-Star Binaries, ApJ 611 (2004) 434.

[21] M. V. Barkov, F. A. Aharonian, S. V. Bogovalov, S. R. Kelner and D. Khangulyan, Rapid TeV Variability in Blazars as a Result of Jet-Star Interaction, ApJ 749 (2012) 119 [1 012 . 1787 ].

[22] V. Bosch-Ramon, M. V. Barkov, D. Khangulyan and M. Perucho, Simulations of stellar/pulsar-wind interaction along one full orbit, A\&A 544 (2012) A59 [1203.5528].

[23] A. Lamberts, S. Fromang, G. Dubus and R. Teyssier, Simulating gamma-ray binaries with a relativistic extension of RAMSES, A\&A $\mathbf{5 6 0}$ (2013) A79 [1309. 7629 ]. 